\title{
Speak before you Think: The Role of Language in Verb Concepts
}

\author{
Tracy PaCKiam AllowaY* and \\ MARTIN CORLEY**
}

\begin{abstract}
This study investigates the role of language on verb concepts in a cross-linguistic environment. The inflectional morphology of verbs in Tamil is contrasted with Mandarin, a language that does not explicitly reflect tense changes in the same manner as Tamil and English. In the baseline condition in experiment 1, participants were required to rate the similarity of object picture pairs; and in the experimental condition, they were asked to rate the similarity of action picture pairs. In experiment 2, participants' similarity judgments and response latencies were recorded when they were presented with the object and action picture pairs. This allowed us to investigate the strength of linguistic influence when representing event concepts. Data from this study revealed a moderate influence of language on verb concepts in the target populations. There was a significant different in the response latencies of the Tamil and Chinese participants, although both language groups exhibited a similar response pattern in the action picture judgment task. This finding suggests that while both groups perceived the events similarly, language may be mediating their responses, resulting in longer response times for the Chinese participants. This could be because considering tense in Mandarin is not 'automatic' and requires extra work, possibly because it is not intrinsic to the verb.
\end{abstract}

\section{Introduction}

Developmental and cross-linguistic research has demonstrated that verbs represent an important relationship between language and knowledge of the world (see Berman \& Slobin, 1994, for a brief review; and Antinucci \& Miller, 1976, for early work in morphology and event construction). One

\footnotetext{
*University of Durham.

** University of Edinburgh.

Correspondence: Dr Tracy Packiam Alloway, Department of Psychology, University of Durham, Science Laboratories, South Road, Durham DH1 3LE, e-mail: t.p.alloway@durham.ac.uk
} 
aspect of this association that has been investigated in great detail is tense markers. Verbal forms vary considerably across languages and linguistic differences in what, when, and how verbs are inflected are evident when comparing narratives produced by different language groups (e.g., Slobin, 1987, 1994).

Cross-linguistic differences in verbal morphology provide the opportunity to explore whether habitually speaking within specific grammatical constraints influences event concepts. The ongoing debate regarding the roles of language and experience in how we think has often been expressed as dichotomous positions: linguistic relativity, the view that linguistic differences lead to variations in conceptual thinking across language groups (e.g., Whorf, 1956; see also Slobin, 1994, for a weaker position); and cognitive determinism, the idea that concepts across different cultures are universal, with different linguistic labels or referents attached to them (e.g., Bloom \& Keil, 2002; Jakendoff, 1990; Macnamara, 1972). Recently however, some researchers have proposed that linguistic effects vary depending on whether a concept is concrete or relational. For example, linguistic effects are less evident for objects, as they are generally identified by perceptually salient features, at least initially. Based on findings from studies on Korean, Mandarin and Italian children, which identified an early concrete noun advantage, despite these languages having more frequent verb input (e.g., Au, Dapretto, \& Song, 1994; Gelman \& Tardiff, 1998), Gentner and Boroditsky (2001) argued that noun partitions tend to follow natural (i.e., perceptual) partitions, leaving the individual to learn linguistic referents for pre-grouped concrete objects.

However, linguistic effects are most visible when there are weak universal or perceptual tendencies (Gentner \& Boroditsky, 2001; Imai \& Gentner, 1997; Munnich, Landau, \& Dosher, 2001). Verbs, for example, are more likely to be learnt from conversations, and thus are more sensitive to syntactic patterns (Choi \& Bowerman, 1991; Gleitman, 1994; Imai \& Mazuka, 1997; Lucy, 1992).

Although there has been some cross-linguistic research on verbal morphology, this has primarily compared narratives of events (e.g., Slobin, 1994). This aim of this study was to explore whether coding differences in verbal morphology lead to variations in thinking about events, particularly in Tamil and Mandarin speakers, the target populations. 


\section{Gharacteristics of Verb Forms}

Verbs are marked by three main grammatical classes - tense, aspect and modality. Tense is the grammatical change to a verb form to illustrate the temporal nature of an action or idea. Aspect is the representation of event structures, which can be expressed by verb semantics. Modal verbs, such as can, do, may, must, ought, should, would, express distinctions of mood, for example, between possibility and actuality. There are other potential morphological markers on verbs, such as number and subjunctivity, however, tense, aspect and modality are potential morphological markers that are commonly considered together because they carry a semantic payload, which affects the quality of the event. In English, tense is expressed by morphological changes to verb forms, however in other languages tense and aspect may be reflected by overt morphology instead. Often times though, tense and aspect are conflated and it is difficult to distinguish between the two. Due to the close relationship between tense and aspect, in this paper, tense refers to morphological changes made to the verb, whereas aspect refers to lexical changes that do not alter the verb form.

The main difference between Mandarin and Tamil verb forms is Mandarin does not have explicit tense markers in the same way that English and Tamil have. In Tamil, tense changes are marked with suffixes added to the root verb (Ardern, 1969; Stever, 1987, 1992). Although there are some irregularities in the past tense forms, tense inflection is simple. In Mandarin, tense changes are generally not marked inflectionally, instead, adverbs may be included to situate the event within temporal parameters. For example, Tomorrow/yesterday/today, he learn the piano. Authors of Mandarin grammar textbooks confirm that Mandarin has a simple verb structure, which according to some linguists gives rise to difficulties when learning a more complex verb system as in English (e.g., Chao, 1968; Waggot, 2000). Table 1 illustrates how English, Tamil and Mandarin speakers express the verb to eat.

When discussing aspect, there are minor differences in usage between Tamil and Mandarin. In Tamil, although the use of aspect markers is optional, a variety of markers can be used to mark different aspectual notions (Renganathan, 1996). It is important to note that the lack of an aspectual marker in a sentence, such as the completive marker (vidu), does not imply that the action is incomplete, simply that the aspectual nature 
Table 1

Present, Past and Future Tense Inflections of the Verb 'to Eat' in English, Tamil and Mandarin

\begin{tabular}{lccc}
\hline Verb - to eat & Present & Past & Future \\
\hline English $\left(3^{\text {rd }}\right.$ person singular $)$ & Eats & Ate & Will eat \\
Tamil $\left(3^{\text {rd }}\right.$ person male $)$ & Saapeda rann & Saapeta an & Saapedu vaan \\
Mandarin & Chi'h & Chi'h & Chi'h \\
\hline
\end{tabular}

of the action is unmarked. In Mandarin, although there is a debate on whether certain adverbs and prepositions are considered as aspect markers due to their positioning within a sentence, Mandarin linguists agree that certain verb changes are marked lexically (see Li \& Thompson, 1981; Tai, 1984; Yeh, 1992).

\section{Present Study}

In this study we investigate whether differences in verbal morphology in Tamil and Mandarin will affect speakers' conceptual representations of event concepts. There are two reasons for the study. The first is to move beyond early research on isolated label differences (e.g., Berlin \& Kay, 1969; Rosch, 1972) and focus on a broader area such as the differences between grammatical patterns. Verb concepts provide a suitable testing ground for this, as there is evidence for the correlation between verbal morphology and event representation (e.g., Huttenlocher, Smiley \& Charney, 1983).

The second motivation for the study is to minimise the interference of cultural and educational factors in cross-linguistic research on the role of language in cognition. In order to do so, both Tamil and Mandarin speakers were recruited from Malaysia, a multi-cultural country where three dominant cultural groups reside amicably. A benefit of conducting testing in Malaysia is that although it is home to different language groups, the relatively small geographical area encourages a fairly homogenous cultural environment. ${ }^{1}$ The Tamil and Mandarin volunteers reside in neighbouring villages, within a 10 to 15 mile radius of each other.

\footnotetext{
${ }^{1}$ Malays are the third dominant cultural group.
} 
Further, both groups also share similar socio-economic backgrounds, thus minimising the disparity in educational levels (see Li \& Gleitman, 2001, for further discussion on the effects of education in cross-linguistic studies). This geographical proximity and cultural similarity reduces any culture-specific anomalies that may occur when recruiting participants from different countries.

In this study, Tamil and Mandarin speakers judged the similarity of picture pairs. Object pairs were used in the baseline condition and action pairs in the experimental condition. The use of the different stimuli for the baseline and experimental conditions was based on the proposal that objects and actions are processed differently, regardless of linguistic variances (cf. Gentner \& Boroditsky, 2001). Objects are directly perceivable and are not strongly influenced by linguistic variances, whereas actions are relational and reliant on linguistic codes (see Sera, Gathje, \& Castillo, 1999, for the argument that linguistic biases in English and Spanish speakers were more likely to influence action categories than object categories). The first experiment was a similarity judgment task, and the second experiment measured response latencies of a same/different decision task.

\section{Experiment 1}

\section{Participants}

Participants were 56 Mandarin and Tamil native speakers from Malaysia - 20 Tamil speakers were tested in the Sekolah Jenis Kebangsan Tamil (local Tamil school), and 36 Mandarin speakers in the San Yuk Mandarin School (local Mandarin school). Participants were 11-12 year olds. For both language groups, their native language was also the dominant language used in their educational environment and home life. Members of neither group spoke English. In addition to the volunteers, there were also four translators who assisted in giving the instructions in either Mandarin or Tamil to the different groups.

\section{Materials}

Two types of stimuli were used in this experiment - object stimuli for a baseline condition and action stimuli for an experimental condition. Both types of stimuli were filmed using a Panasonic EZ1 mini television camera. 
The images were then fed into a Macintosh computer and recorded using an Apple Video Player to capture them as stills. ${ }^{2}$ All images were taken in the same room with the same background and lighting conditions. For the baseline condition, objects were filmed on a solid blue background. For the experimental condition, actions were filmed against a brown wall. All images were then saved as 'bitmap' conversions, printed, laminated and mounted on $7.5 \mathrm{~cm} \times 10 \mathrm{~cm}\left(3^{\prime \prime} \times 4^{\prime \prime}\right)$ cards.

In the baseline condition, photographs of different mugs were the test items, and various other items, such as keys and books, were fillers. There were 9 pairs of test stimuli consisting of a Base picture and an Object Change picture. The Base form was a photograph of a mug, and the Object Change form depicted an alternation of the same mug, for example, the handle was broken. There were 11 filler stimuli of unrelated items, such as keys, a book, and a hammer. These items were only presented in their original condition. The object stimuli were paired in the following conditions and are depicted in Figure 1: same condition - the Base form of a mug was presented with the corresponding Object Change form; same type condition - two different mugs were shown; and different condition unrelated items were paired together, such as a book and a set of keys.

In the experimental condition, there were 20 picture sets represented as triplets of Base, Action Change and Theme Change forms (B, AC, TC). The Base form (pic B) showed the agent in the process of committing an action, for example a lady kicking a ball, or a lady poking another lady. The Action Change form (pic AC) depicted the same action at the point of completion, for example, the man kicked the ball (i.e., the ball is in the air); or the lady poked another lady. The Theme Change form (pic TC) showed the same action as the base form, but the theme was changed, for example, the lady kicks a poster tube, rather than a ball; or the lady pokes a man instead of a lady. Of the 20 sets of pictures, 10 showed inanimate themes (e.g., ball/poster tube) and 10 showed animate themes (e.g., lady/man).

The action stimuli were paired in the following combinations and are depicted in Figure 2: the B-AC combination represented the same agent, performing the same action on an inanimate object, but at a different

\footnotetext{
${ }^{2}$ The photos were taken using a television camera instead of a digital camera to allow for flexibility in capturing the appropriate action sequences. For consistency, the objects for the baseline condition were also shot with a television camera.
} 

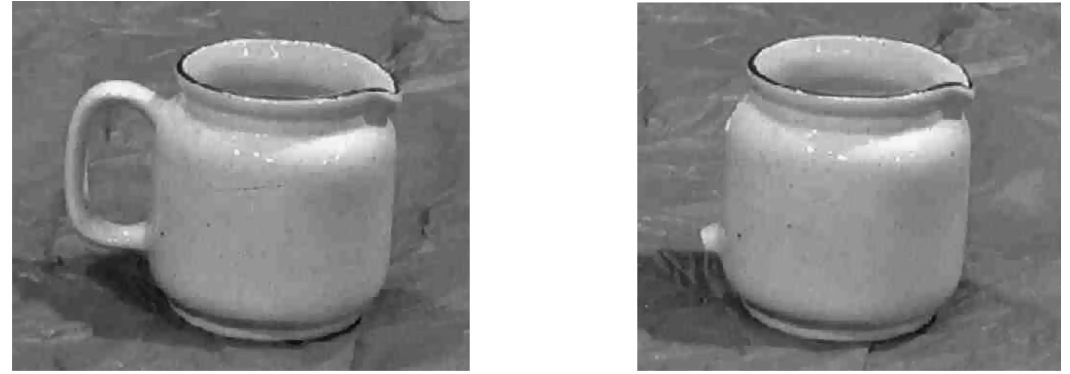

Same combination: same object, different form
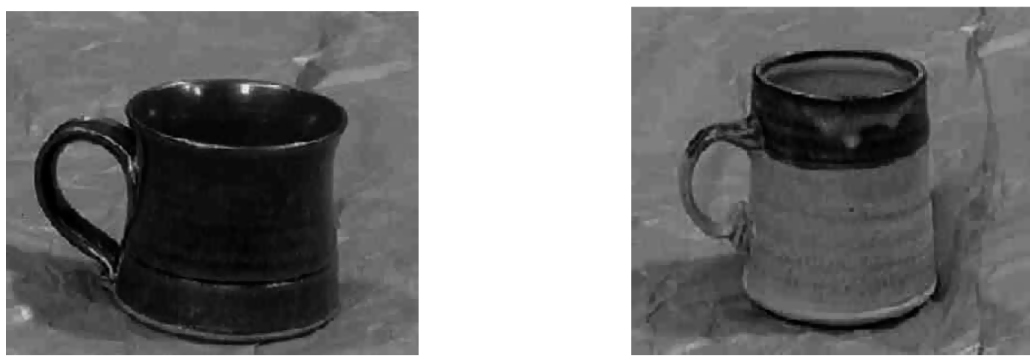

Same type combination: different object, same form
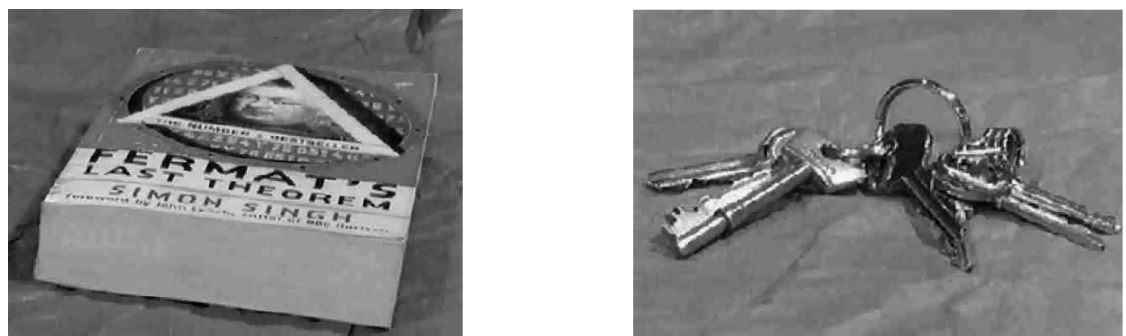

Different combination: different object, different form

Figure 1. Different picture combination pairs in the baseline condition.

stage, for example, a lady kicks/kicked a ball; in the B-TC combination, the same agent, executed the same action, at the same point in time, on a different object/person, for example, a lady kicks a ball/poster tube; the filler pair depicted different people, doing different actions, at the same point in time, for example, a man punching another man was paired with a picture of a lady giving something to a man. 

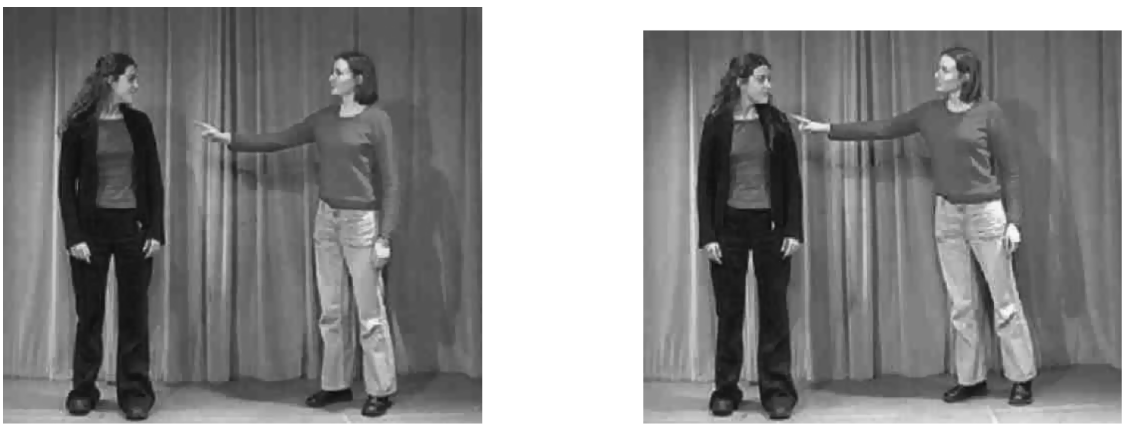

B-AC combination: same agent, different tense, same theme
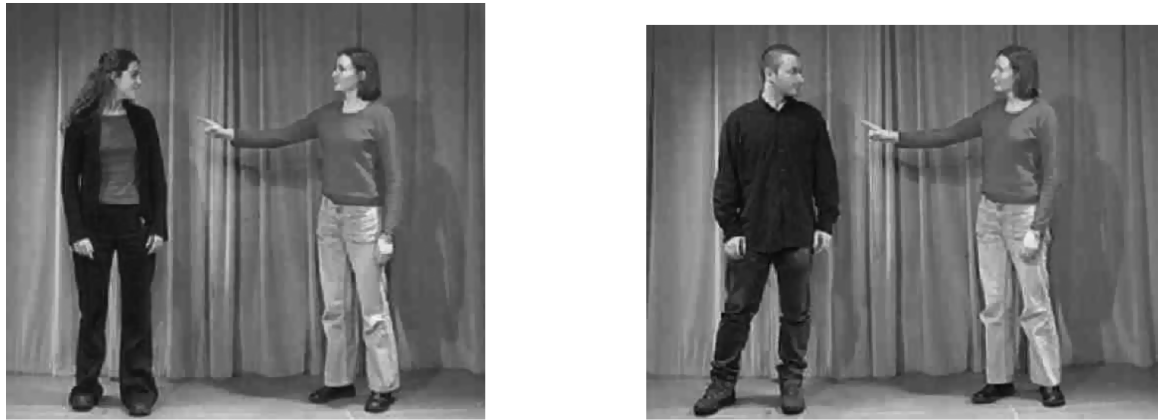

B-TC combination: same agent, same tense, different theme
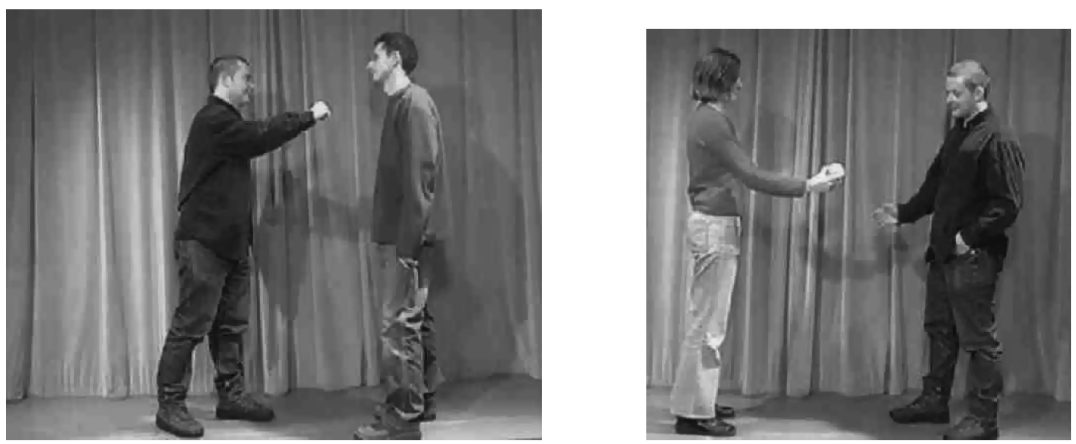

Filler combination: different agent, same tense, different theme

Figure 2. Different picture combination pairs with animate themes in the experimental condition. 
The base picture served as a reference for the subsequent picture (either Action Change or Theme Change). The B-AC picture pairs reflected tense differences as the $\mathrm{AC}$ picture depicted the completion of the action in the Base picture. Tamil speakers, like English speakers, use past tense markers to express this difference, for example, the lady kicks the ball; the lady kicked the ball. The Mandarin speakers however, are not obligated to express this difference grammatically. The B-TC picture pairs did not invoke any tense-aspectual forms because the TC picture showed the same action (at the same stage) and the same agent as the Base picture. The only difference was the theme - inanimate/animate - in the picture. The B-TC combination was a control to ensure that participants were focusing on the action, rather than the person performing the action. Thus, both Tamil and Mandarin participants should rate the actions in the B-TC pair as more similar than the B-AC pairs.

All pictures in the study depicted dynamic events that included a resultative state rather than a stative one (i.e., to drop, to push, to hit, rather than to know, to think), and the B-AC pictures represented punctual events. To accommodate the completive and progressive markers available in both Mandarin and Tamil, the action in the picture pairs was limited to these two forms - completed (B-AG) and progressive (B-TC).

\section{Procedure}

There were two conditions: a baseline condition and an experimental condition. In the baseline condition, participants were shown 20 pairs of object stimuli - 3 sample stimuli, 6 same pair stimuli, 6 same type pair stimuli, and 5 different pair stimuli. In the experimental condition, there were 55 pairs of action stimuli - 3 sample stimuli, 20 B-AC pairs, 20 B-TC pairs and 12 filler pairs. Stimuli presentation was counterbalanced to minimise order effects. Participants received the following instructions through a translator: Rate how similar you think these objects/actions are. They were instructed to circle a number between 1 (not similar) and 7 (very similar) on the response sheet to represent the similarity of the object pairs. Participants were given a short break between the baseline and experimental conditions. 
Hypotheses

Baseline Condition

Based on the proposal that objects are perceptually grounded and not affected by labelling differences (cf. Gentner \& Boroditsky, 2001), it was anticipated that the Tamil and Mandarin speakers would exhibit similar response patterns. However, as the objects were paired in such a way that certain pairs were more similar (e.g., two mugs in the same and same type conditions) than others (e.g., a book and a hammer in the different condition), the picture pairs should be rated differently from each other.

Experimental Condition

In the experimental condition, differences in expressing events can be explicated in one of two ways.

Language-as-an-infuence hypothesis (Hypothesis LI)

The premise of this hypothesis is that verbs are more relational than perceptual, consequently linguistic effects will be evident. It was anticipated that Tamil speakers' similarity judgments should reflect the marked tense structure, and the actions in the B-AC pairs will be rated as less similar compared to the B-TC pairs. The Mandarin speakers, who do not have a marked tense structure, will be influenced accordingly, and rate the actions in both the B-AC and B-TC picture pairs similarly.

Language-as-a-reflection hypothesis (Hypothesis LR)

According to this view, speakers of different languages share commonalities in how they think, and are not influenced by linguistic structure. Consequently, Tamil and Mandarin speakers share a similar representation of events and should exhibit a similar pattern of ratings for the B-AC and B-TC picture pairs.

Results and Discussion

Baseline Condition

Similarity scores for the three conditions for Picture type - same, same type and different - were averaged (with standard deviations in parentheses): Tamil participants: 4.3 (1.6); 3.4 (1.1); 2.2 (1); Mandarin participants: 5.3 


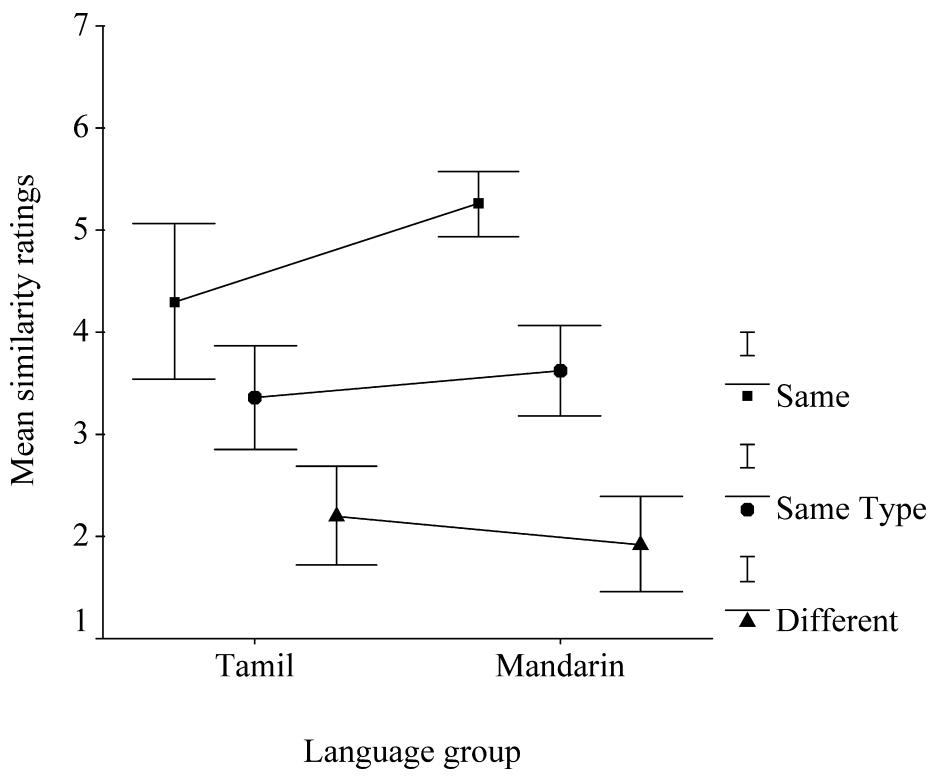

Figure 3. Mean ratings $(1=$ not similar; $7=$ similar $)$ for picture similarity for Object pairs (same, same type and different), by linguistic group of participants.

(.9); 3.6 (1.3); 1.9 (1.4) for each of the picture conditions respectively (see Figure 3). The mean similarity ratings appear to be comparable across the two language groups.

A two-way mixed design ANOVA with language groups and picture pairs $\left(F_{1}\right.$; Language $=$ between, Picture $=$ within; $F_{2}$; Language $=$ within, Picture $=$ between) as random factors and similarity judgments as the dependent measure, confirmed the hypothesis that Tamil and Mandarin participants responded similarly to the object pairs. There was no difference in the similarity ratings between the two language groups, $F_{1}(1,53)=1.20, p=.27 ; F_{2}(1,14)=2.97, p=.11$. There was a main effect for the Picture type, $F_{1}(2,106)=77.30, p<.001 ; F_{2}(2,14)=34.05$, $p<.001$. This difference is expected, as certain pairs were more similar than others, such as two types of mugs compared to a set of keys and a book. The interaction between the two factors was not significant, $F_{1}(2,106)=2.30, p=.11 ; F_{2}(2,14)=2.99, p=.08$. Post-hoc Sheffe's tests confirm the difference in similarity ratings between object pairs, as all factors were rated significantly different from each other (all pairwise 


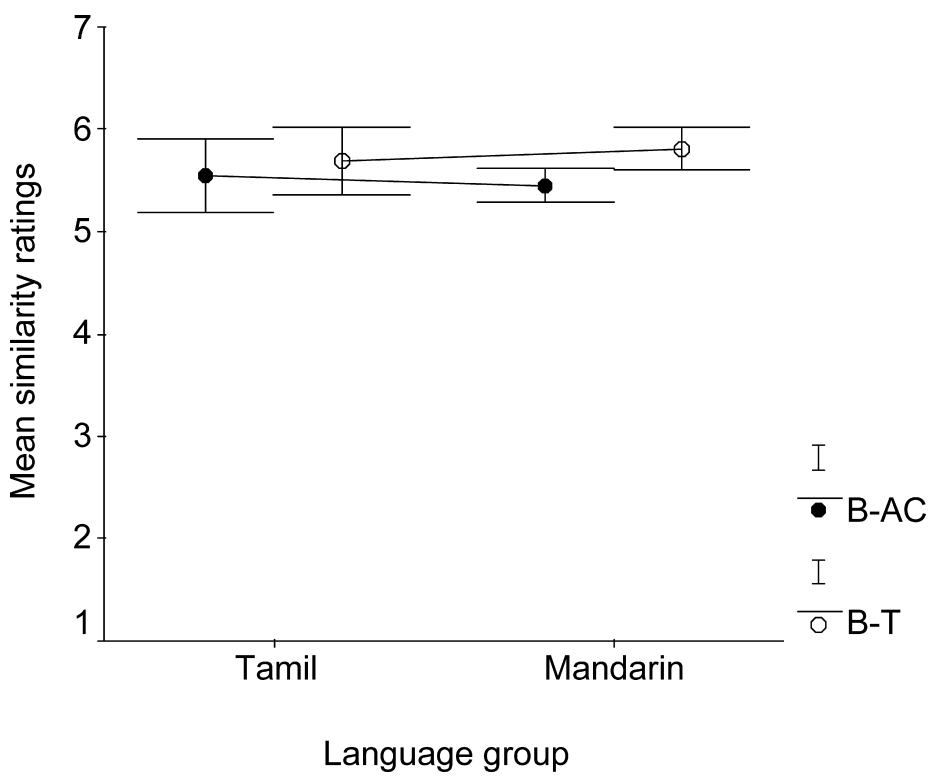

Figure 4. Mean ratings $(1=$ not similar; $7=$ similar $)$ for picture similarity for Action Change (B-AG) and Theme Change (B-TG) pairs, by linguistic group of participants.

comparisons were significant at $p<.05)$. Overall, the findings from the baseline condition support the hypothesis and suggest that both language groups interpreted the instructions similarly.

\section{Experimental Condition}

In the experimental condition, although the mean ratings for the $\mathrm{B}-\mathrm{AC}$ pairs are slightly lower than for the B-TC pairs (with standard deviations in parentheses): B-AC - 5.55 (.8) and 5.45 (.5); B-TC - 5.7 (.7) and 5.8 (.6) by Tamil and Mandarin participants respectively, there does not appear to be any difference in similarity judgments between the two language groups (see Figure 4).

A two-way mixed design ANOVA indicated that both language groups responded similarly to the picture pairs, $F_{1}(1,54)=0.01, p=.92$; $F_{2}(1,38)=0.04, p=.85$. The by-participants analyses show that the $\mathrm{B}-\mathrm{AC}$ and B-TG picture pairs were rated differently, $F_{1}(1,54)=8.20$, $p=.01$, however this was not confirmed in the by-items analyses, 
$F_{2}(1,38)=3.13, p=.09$. The interaction between the two factors was not significant, $F_{1}(1,54)=1.65, p=.20 ; F_{2}(1,38)=1.17, p=.20$. The results do not provide any support for the influence of language on cognition. Instead, linguistic variances did not appear to affect the similarity ratings, and both Tamil and Mandarin speakers produced a similar pattern of similarity ratings.

There are some concerns with this experiment. It is possible that the Mandarin speakers might interpret the similarity scales differently from the Tamil speakers, and the similarity ratings might be an artefact of the task. Other methodological frameworks might be more effective in assessing more subtle linguistic contributions to event concepts. In light of this possibility, the following experiment investigated on-line processes of a same/different judgment task measured by response latencies (see Morrow, Bower, \& Greenspan, 1990, for a similar methodology in research in narrative comprehension). Decision tasks elicit a speeded response from a participant and are a common methodological tool in psycholinguistic research (see Gernsbacher, 1994, for a review of various studies). The response latency is thought to be reflective of an explicit and inferred activation of the target information. For example, a faster response indicates quick activation of a verb concept, whereas a slower response might be the result of conflicting grammatical and perceptual information for action pictures. The pattern of response latencies allows a researcher to infer the nature of verb structure and its effect on the speaker, and by comparing the speed of response to the B-TC pairs with the B-AC pairs, we can explore the influence of verb structure on event concepts (see Gernsbacher, 1990; Ratcliff \& McKoon, 1981, for psycholinguistic research using response latencies in decision tasks).

\section{Experiment 2}

\section{Participants}

There were 60 participants, 30 Tamil speakers and 30 Mandarin speakers. Both language groups had approximately equal numbers of 11-12 years old and adult speakers. The Tamil children were attending the Sekolah Jenis Kebangsan Tamil (local Tamil school), and the Mandarin children were students at the San Yuk Mandarin School. The adults were residents of a small local community recruited from the same villages as participants 
from experiment 1. Both language groups resided in Malaysia, and their native language was the dominant language, used in school and at home. Members of neither group spoke English or had volunteered for the previous experiment.

\section{Materials}

The materials were the same object and action stimuli used in Experiment 1. All pictures were presented on a $21 \mathrm{~cm}$ by $28 \mathrm{~cm}\left(8^{\prime \prime} \times 11^{\prime \prime}\right)$ coloured screen of a PowerBook Macintosh laptop, using Psyscope software (Cohen, MacWhinney, Flatt, \& Provost, 1993).

\section{Procedure}

Each participant was tested individually. They were seated in front of a portable PowerBook Macintosh computer and asked to read the following instructions written in their language: You will see two pictures. I would like you to look at these two pictures and tell me if you think these two pictures are the same type of object (action). If you think they are the same type of object (action), press the button that says 'SAME.' If you think they are not the same type of object (action), press the button that says 'DIFFERENT.'

The pictures in both the baseline and experimental conditions were paired in the same manner as described in the previous experiment. The picture pairs were presented on a laptop computer in a randomised order. Participants were instructed to press a key indicating whether they considered the pictures as the same (key $P$ ) or different (key Q). The corresponding keys were on separate sides of the keyboard to minimise the probability of pressing the wrong key. Participants were allowed to use whichever hand they were most comfortable with, although most used both hands. The stimuli remained on the screen until either key $P$ or $Q$ was pressed. The computer program recorded the participants' responses (i.e., same or different) and response time (in milliseconds) to the picture pairs.

\section{Hypotheses}

\section{Baseline Condition}

The first prediction for this condition is that the Tamil and Mandarin speakers should produce similar responses to the different object pairs. 
Table 2

Summary of Predictions for Tamil and Mandarin Speakers in the Experimental Condition

\begin{tabular}{lcc}
\hline Hypotheses & $\begin{array}{c}\text { Similar response patterns } \\
\text { (same vs. different) }\end{array}$ & $\begin{array}{c}\text { Similar response latencies } \\
\text { (in msec) }\end{array}$ \\
\hline Hypothesis LI $\mathrm{LI}_{1}$ & $\mathrm{X}$ & $\mathrm{X}$ \\
Hypothesis $\mathrm{LI}_{2}$ & $\mathrm{X}$ & $\mathrm{X}$ \\
Hypothesis LR & & \\
\hline
\end{tabular}

Because some of the object pairs consist of noticeably different items, there should be a difference in how they are rated, for example, participants will be more likely to respond same to photos of two mugs in the same and same type condition than a book and a hammer in the different condition. However, the Mandarin speakers' responses to the object pairs will not differ from the Tamil speakers' responses. Secondly, Tamil and Mandarin speakers should spend comparable amounts of time responding to the object pairs, as reflected by their response latencies.

Experimental Condition

We can investigate the strength of linguistic influences in the experimental condition by examining the similarity judgments and response latencies. The predictions are summarised in Table 2 .

\section{Language-as-a-strong-influence hypothesis (Hypothesis $L I_{1}$ )}

This premise is that linguistic patterns are the dominant conceptual patterns that influence cognition, often precluding alternative representations. There are two predictions according to this hypothesis. First, the Tamil and Mandarin speakers' responses will reflect the corresponding verb characteristics. Specifically, the explicitly marked verb structure in the Tamil language will direct speakers to consider the B-AC picture pairs as different and the B-TC pairs as the same. Because of the relatively simple verb structure in Mandarin, speakers will consider both B-AC and B-TC picture pairs as the same.

The second prediction is that the language groups will not differ in the amount of time they spend judging the similarity of the actions (measured by response latencies). Since linguistic patterns have been ingrained during 
language learning, conceptual parameters are automatised and therefore, speakers do not need to explicitly access language while performing the task. Although language is influencing the Tamil and Mandarin speakers' conceptualisation of events, it does not directly mediate their responses during the task.

\section{Language-as-a-weak-influence hypothesis (Hypothesis $L I_{2}$ )}

Proponents of this hypothesis argue that although Mandarin speakers express events differently from Tamil speakers, this does not preclude them from conceptualising them similarly. As a result, the first prediction is that both Tamil and Mandarin speakers will produce similar response patterns to the action pairs.

Based on the premise that participants are accessing language when performing the task, the second prediction is that Tamil and Mandarin speakers should differ in how long they spend in decision-making. In particular, Mandarin speakers should take a longer time to respond to the B-AC action pairs (same action, different tense) because the implicit tense structure may require extra work. The Tamil speakers however, will not face such decision-making conflict, and will respond more quickly to the B-AC picture pairs compared to the Mandarin speakers.

\section{Language-as-a-reflection hypothesis (Hypothesis LR)}

The prediction in this position is based on the idea that linguistic differences when expressing events do not ultimately influence speakers' conceptual representations of them. Therefore, Tamil and Mandarin participants should produce similar response patterns in their similarity judgments, and their deliberation of the picture pairs will not be significantly different.

\section{Results and Discussion}

For the baseline and experimental conditions, analyses were conducted with participants $\left(F_{1}\right.$; Language $=$ between, Picture $=$ within $)$ and items $\left(F_{2}\right.$; Language $=$ within, Picture $=$ between $)$ as random factors, on the following dependent measures: response latencies (measured in milliseconds), response index (based on the categorical choice of same and different), and response latencies by response patterns (same vs. different). 
Table 3

Mean Response Latencies (in msecs) for Object Pairs by Language Group

\begin{tabular}{lccc}
\hline Language group & \multicolumn{3}{c}{ Picture combinations } \\
\cline { 2 - 4 } & Same & Same type & Different \\
\hline Tamil & $2872(1273)$ & $3859(3268)$ & $3351(1926)$ \\
Mandarin & $3876(2539)$ & $3832(2379)$ & $3933(2748)$ \\
\hline
\end{tabular}

Note. Standard deviations are in parentheses.

\section{Baseline Condition}

Response Latencies

The mean response latencies of the Tamil and Mandarin speakers suggest that they did not differ much in their response times for each object pair (see Table 3). In a two-way mixed design ANOVA, by-participants analyses revealed no language effects, $F_{1}(1,58)=0.9 ; p=.35$; there was however a small effect for language group in the by-items analyses; $F_{2}(1,14)=5.4, p=.04$. There was no main effect in response times for the different pictures, $F_{1}(2,116)=1.57 ; p=.22 ; F_{2}(2,14)=0.39$, $p=.68$. There was also no interaction between language group and picture pairs, $F_{1}(2,116)=1.82 ; p=.16 ; F_{2}(2,14)=1.9, p=.18$. The non-significant interaction confirms that Tamil and Mandarin speakers spent comparable time deliberating their responses.

Response Patterns

In order to analyse the responses produced by the participants, a response index based on the separate totals for same and different responses, was calculated in order to compare responses for B-AC and B-TC pictures. For the $\mathrm{B}-\mathrm{AC}$ picture pairs, the formula for the index was $\mathrm{B}-\mathrm{AC}$ same/(B-AC same + B-AC different). The same calculation was used for the B-TC pictures, with responses to the B-TC pairs. In the response index calculations, the closer the figure is to 1, the more same responses the participant produced. If the figure is closer to 0 , the participant tended to produce more different responses. The response index means are summarised in Table 4 and indicate that although the Mandarin speakers were more likely to respond same to object pairs in the same and same type condition, responses in both language groups decreased monotonically across conditions. 
Table 4

Mean Response Index (0-1) for Object Pairs by Language Group

\begin{tabular}{lccc}
\hline Language & \multicolumn{3}{c}{ Picture combinations } \\
\cline { 2 - 4 } & Same & Same type & Different \\
\hline Tamil & $.65(.24)$ & $.43(.24)$ & $.03(.09)$ \\
Mandarin & $.84(.19)$ & $.74(.27)$ & $.05(.1)$ \\
\hline
\end{tabular}

Note. Standard deviations are in parentheses; scores closer to 1 indicate that the participant produced more same responses, and scores closer to 0, indicate more different responses.

A mixed two-way ANOVA was conducted with the response index as the dependent variable. There was a main effect for language groups; $F_{1}(1,58)=20.8, p<.001 ; F_{2}(1,14)=24, p<.001$. There was also a significant difference between picture pairs; $F_{1}(2,116)=308.96$, $p<.001 ; F_{2}(2,14)=29.5, p<.001$. The interaction was reliable; $F_{1}(2,116)=12.4, p<.001 ; F_{2}(2,14)=5.5, p=.02$. Scheffe's post hoc analysis yielded statistically significant differences $(p<.05)$ between same and different picture pairs, and between same type and different picture pairs. These combinations were also significant in paired sample t-tests with criterion adjusted to $p<.0167$, in addition the responses to the same and same type pictures were also significant.

From the mean responses, the Mandarin speakers appear to have judged the same and same type object pairs as same more frequently than the Tamil speakers. One suggestion is that the Mandarin participants have a natural bias towards judging picture pairs as same, for example, in the baseline condition in experiment 1 , they also produced higher similarity ratings than Tamil speakers. The increased frequency of same responses could be a task artefact, rather than language-specific responses. In light of this possibility, it may be more productive to look at on-line processes such as response latencies in this experiment. Although differences between language groups in interpreting similarity tasks are expected, it is important to note that the Tamil and Mandarin speakers' response patterns to the object pairs were similar, as the responses increased monotonically across object conditions. 
Response Latencies by Response

Some researchers who measure reaction time in decision task have suggested that it takes longer to judge a false description than a true one (e.g., Clark \& Chase, 1972, 1974). In light of the fact that there are different cognitive processes that underlie same and different responses (or true/false responses), response latencies when producing same and different responses for the three picture conditions - same, same type and different picture pairs were analysed separately.

However, as some participants responded to all the stimuli in a condition as same or different (e.g., participant 2 responded to all sametype items as the same; participant 13 perceived all B-AC items as different), there were inevitably empty cells. In order to preserve a balanced design with minimal agitation to the observed variances, the following formula was used to estimate the missing cells means:

$$
\mathrm{AB}_{\mathrm{ij}}^{\prime}=\mathrm{A}_{\mathrm{i}}^{\prime}+\mathrm{B}_{\mathrm{j}}^{\prime}-\mathrm{G}^{\prime} \quad \text { (Winer, 1971, p. 488) }
$$

Missing cell means $=$ Subject mean $($ or Item mean $)+$ Condition mean Grand mean

The mean response times by responses are displayed in Table 5 . The Mandarin speakers' mean response latencies are slightly higher than the Tamil speakers' response times and can be explained when looking at the raw data for items. For example, a few Mandarin speakers responded same to some of the object pairs in the different category and spent a long time deliberating over this choice. This response is unexpected as the object pairs in the different category were clearly different, for example, a hammer and a book. Their longer response times suggests that they may have been searching for any commonality between the object pictures in order to judge them as the same (in the above example, a commonality might be that they are both kept in the house).

In the first analysis, comparing same responses across the three picture conditions, there was no main effect for Language group in the byparticipants analyses, $F_{1}(1,58)=1.7, p=.19$; however, there was a significant difference in the Tamil and Mandarin speakers' responses in the by-items analyses, $F_{2}(1,14)=9.2, p=.009$. In the by-participants analyses, there was a main effect for Picture type, $F_{1}(2,116)=116.5$, $p<.001$; but not in the by-items analyses, $F_{2}(2,14)=0.93, p=.42$. The interaction between the two factors was not significant, $F_{1}(2,116)=0.41$, 
Table 5

Mean Response Latencies (in msec) as a Function of Participants' Responses for Object Pairs

\begin{tabular}{lcccccc}
\hline Language & \multicolumn{6}{c}{ Picture combinations by response } \\
\cline { 2 - 7 } & $\begin{array}{c}\text { Same/ } \\
\text { Same }\end{array}$ & $\begin{array}{c}\text { Same/ } \\
\text { Different }\end{array}$ & $\begin{array}{c}\text { Same type/ } \\
\text { Same }\end{array}$ & $\begin{array}{c}\text { Same type/ } \\
\text { Different }\end{array}$ & $\begin{array}{c}\text { Different/ } \\
\text { Same }\end{array}$ & $\begin{array}{c}\text { Different/ } \\
\text { Different }\end{array}$ \\
\hline Tamil & $3484(2185)$ & $4408(5330)$ & $3327(1987)$ & $3243(1921)$ & $6797(2393)$ & $2869(1284)$ \\
Mandarin & $4033(2567)$ & $4153(2549)$ & $4339(3702)$ & $3558(2435)$ & $7647(2618)$ & $3708(2304)$ \\
\hline
\end{tabular}

Note. Standard deviations are in parentheses.

$p=.67 ; F_{2}(2,14)=2.3, p=.13$, indicating that both language groups spent similar amounts of time when judging the picture pairs as the same.

In the second analysis, with different responses, there was no main effect for Language group, $F_{1}(1,58)=0.23, p=.63 ; F_{2}(1,14)=0.13, p=.73$. In the by-participants analyses, there was a significant main effect for Picture type, $F_{1}(2,116)=4.2, p=.02$; but it was not confirmed in the by-items analyses, $F_{2}(2,14)=1.6, p=.23$. The interaction between the two factors was not significant, $F_{1}(2,116)=1.07, p=.35 ; F_{2}(2,14)=1.3$, $p=.31$, confirming that the length of time both language groups spent deliberating over the object pairs when they judged them to be different was comparable. Overall, the findings from the same and different responses are expected and indicate that the Tamil and Mandarin speakers took similar amounts of time in deliberating over their responses to the object pairs.

\section{Experimental condition}

Reponses Latencies

The mean response latencies for the Tamil and Mandarin participants are presented in Table 6. The Mandarin speakers took longer than the Tamil speakers to respond to both picture conditions. Moreover, the Mandarin speakers took twice as long to respond to the B-AC pairs compared to the B-TC pairs (a 1182 vs. a 566 msec difference from Tamil speakers' response times). Analyses from a two-way mixed design ANOVA indicated that response latencies between Tamil and Mandarin speakers differed significantly, $F_{1}(1,58)=3.7, p=.05 ; F_{2}(1,38)=46.15, p<.001$. 
Table 6

Mean Response Latencies (in msecs) for Action Pairs by Language Group

\begin{tabular}{lcc}
\hline Language & \multicolumn{3}{c}{ Picture combinations } \\
\cline { 2 - 3 } & B-AC & B-TC \\
\hline Tamil & $3336(1205)$ & $3235(1244)$ \\
Mandarin & $4518(2762)$ & $3801(1876)$ \\
\hline
\end{tabular}

Note. Standard deviations are in parentheses.

Table 7

Mean Response Index (0-1) for Action Pairs by Language Group

\begin{tabular}{lcc}
\hline Language & \multicolumn{3}{c}{ Picture combinations } \\
\cline { 2 - 3 } & B-AC & B-TC \\
\hline Tamil & $0.71(.25)$ & $0.81(.23)$ \\
Mandarin & $0.64(.23)$ & $0.84(.14)$ \\
\hline
\end{tabular}

Note. Standard deviations are in parentheses; scores closer to 1 indicate that the participant produced more same responses, and scores closer to 0 , indicate more different responses.

There was also a significant difference for response times of the picture pairs; $F_{1}(1,58)=5.56, p=.02 ; F_{2}(1,38)=5.77, p=.02$. In the by-participants analyses, although the interaction between the two factors narrowly missed significance; $F_{1}(1,58)=3.2, \mathrm{p}=.07$; there was a reliable interaction in the by-items analyses, $F_{2}(1,38)=5.78, p=.02$. This finding provides some support for Hypothesis $\mathrm{LI}_{2}$, as the difference in verb structure between the two language groups affected their decision-making time.

\section{Response Patterns}

Summarised in Table 7 are the mean responses (calculated by the response index described above) of the Tamil and Mandarin speakers. Scores closer to 1 signify more same responses, whereas scores nearer 0 denote more different responses. The response index means suggest that both language groups exhibited similar response patterns to the picture pairs, and both language groups judged the $\mathrm{B}-\mathrm{AC}$ pairs to as different compared to the B-TC pairs. 
Table 8

Mean Response Latencies (in msec) as a Function of Participants' Responses for Action Pairs

\begin{tabular}{llccr}
\hline Language & \multicolumn{4}{c}{ Picture combinations by response } \\
\cline { 2 - 5 } & B-AC/Same & B-AC/Different & B-TC/Same & B-TC/Different \\
\hline Tamil & $3354(1213)$ & $3828(2471)$ & $3298(1303)$ & $3398(1853)$ \\
Mandarin & $4260(2698)$ & $5250(3522)$ & $3722(1814)$ & $4005(2335)$ \\
\hline
\end{tabular}

Note. Standard deviations are in parentheses.

A two-way mixed design ANOVA indicated no main effect for Language groups, $F_{1}(1,58)=0.27, p=.70 ; F_{2}(1,38)=0.63, p=.40$. There was a main effect for Picture pairs, $F_{1}(1,58)=23.9, p<.001$; $F_{2}(1,38)=6.8, p=.01$. In the by-participants analyses, the interaction between variables was not significant, $F_{1}(1,58)=2.7, p=.11$; however it was significant in the by-items analyses, $F_{2}(1,38)=5.7, p=.02$. In light of this finding, it is difficult to argue for strong support for Hypothesis $\mathrm{LI}_{1}$, as the different verb structures did not appear to influence how the Tamil and Mandarin speakers judged the action combinations.

Response Latencies by Response

From the response latency means (see Table 8), the Tamil speakers response times appear to be stable across picture conditions and responses. The Mandarin volunteers however, seemed to take considerably longer when responding different, particularly in the B-AC picture condition (compare the difference in mean response latencies: a $1422 \mathrm{msec}$ difference for the B-AC pictures; and a 607 msec difference for the B-TC pictures).

The Tamil and Mandarin speakers' response latencies were analysed separately according to responses produced. First, for the same response, analyses from a two-way mixed design ANOVA indicated no main effect for language groups in the by-participants analyses, $F_{1}(1,58)=2.3$, $p=.14$; however, in the by-items analyses, the language groups differed significantly, $F_{2}(1,38)=22.6, p<.001$. There was no main effect for picture pairs, $F_{1}(1,58)=2.4, p=.13 ; F_{2}(1,38)=2.9, p=.10$. The interaction between variables was not significant either; $F_{1}(1,58)=1.6$, p $=.22 ; F_{2}(1,38)=3.3, p=.07$. 
For the different responses, the difference in response times between language groups was nearly significant in the by-participants analyses, $F_{1}(1,58)=3.3, p=.07$; and was reliable in the by-items analyses, $F_{2}(1,38)=19.3, p<.001$. In the by-participants analyses, there was a main effect for picture pair, $F_{1}(1,58)=4.7, p=.03$; however, this was not confirmed in the by-items analyses, $F_{2}(1,38)=0.001, p=.98$. The interaction was not significant, $F_{1}(1,58)=1.12, p=.30 ; F_{2}(1,38)=0.04$, $p=.84$. In this instance, there is weak evidence for the predictions in Hypothesis LI2, as the Mandarin speakers' response times to the B-AC pairs were significantly longer than to the B-TC pairs.

The results in the experimental condition do not support Hypothesis LR, as tense morphology influenced representations of event concepts. In particular, Tamil and Mandarin speakers differed in how long they spent judging the actions in the picture pairs as same or different. Thus, while representations of more perceptually grounded concepts, such as objects, are perhaps universal, other concepts such as events, appear to be affected by grammatical differences.

\section{General Discussion}

When reviewing the results from all the experiments in this study, we can make the following suggestions regarding the role of language. The findings from experiment 1 suggest that Mandarin speakers are likely to use tensemarkers, despite their optionality. The results in experiment 2 allow us to surmise two things. First, verb differences did not affect the actual responses produced by the Tamil and Mandarin speakers. This result corresponds with the pattern of similarity ratings produced in experiment 2 , and suggests that language does not play a strong role in influencing conceptual representations. However, the Tamil and Mandarin speakers produced different response times, in particular, the Mandarin speakers spent longer deliberating over the B-AC picture pairs when responding to them as different. This could be because considering tense in Mandarin is not 'automatic' and requires extra work, possibly because it is not intrinsic to the verb. This finding is in-line with the proposal made by Hunt and Agnoli (1991) that having a direct lexical entry for a concept speeds up speakers' processing time. 
Although language appears to play some role, albeit a weak one, in how concepts are represented, we can further explore its influence. For example, the study did not directly investigate whether language intervenes during the processing of picture pairs. The difference between the influence of linguistic structure on representations while speaking but not otherwise, or if grammatical patterns exert any influence on representations even when language is not accessed as a result of pre-set conceptual parameters, requires further study before making further claims regarding the role of language in cognition. A future study examining this distinction would require an additional group of Tamil and Mandarin participants to articulate nonsense sounds that are phonologically compatible in both languages during the task in order to load their verbal memory (see Gennari, Sloman, Malt \& Fitch, 2002, for a similar strategy). The use of articulartory suppression would prohibit participants from effectively accessing language, allowing a detailed comparison of findings from participants who are allowed to access language with those who are not. This particular design would provide insight to whether event concepts are pre-set during the language-learning stage with no further interference from language, or if the role of language is more dynamic, providing constant input to the conceptual and perceptual processes.

The study investigated whether speakers of Mandarin, a language that does not have explicit tense morphology, would conceptualise events differently from speakers of Tamil, a language those who do, such as. The findings suggest that differences in verbal morphology do not necessarily influence how events are conceptualised, however, they can affect how quickly the concepts are accessed.

\section{REFERENCES}

Antinucai, F. AND R. Miller

1976 How Children Talk about What Happened. Journal of Child Language 3, 167-89. ARDEN, A.H

1942, reprinted 1969 A Progressive Grammar of the Tamil Language. Madras, India: Christian Literature Society.

AU, T.K., M. Dapretto And Y.K. Song

1994 Input vs. constraints: Early word acquisition in Korean and English. Fournal of Memory and Language 33, 567-582. 
BERLIN, B. AND P. KAY

1969 Basic Color Terms: Their Universality and Evolution. Berkeley, CA: University of California.

BERMAN, R.A. AND D.I. SLOBin

1994 Relating Events in Narrative: A Crosslinguistic Developmental Study. Hillsdale, NJ: Lawrence Erlbaum Associates.

BLOOM, P. AND F. KEIL

2001 Thinking through language. Mind \& Language 16, 351-367.

CHAO, Y.-R.

1968 A Grammar of Spoken Chinese. California: University of California Press.

CHOI, S. AND M. BOWERMAN

1991 Learning to express motion events in English and Korean: The influence of language-specific lexicalization patterns. Cognition 41, 83-121.

Cohen, J.D., B. MacWhinney, M. Flatt and J. Provost

1993 PsyScope: A new graphic interactive environment for designing psychology experiments. Behavioral Research Methods, Instruments, and Computers 25(2), 257-271.

Gelman, S.A. AND T. TARdiF

1998 A cross-linguistic comparison of generic noun phrases in English and Mandarin. Cognition 66(3), 215-248.

Gennari, S., S.A. Sloman, B.C. Malt and W.T. Fitch

2002 Motion events in language and cognition. Cognition 83, 49-79.

GentNer, D. AND L. BORODITSKY

2001 "Individuation, Relativity and Early Word Learning." In M. Bowerman and S. Levinson (Eds.), Language Acquisition and Conceptual Development (pp. 215-256). New York: Cambridge University Press.

Gernsbacher, M.A.

1990 Language Comprehension as Structure Building. Hillsdale, NJ: Erlbaum.

1994 (Ed.) Handbook of Psycholinguistics. San Diego, CA: Academic Press.

Hunt, E. AND F. AGNOLI

1991 The Worfian hypothesis: A cognitive psychology perspective. Psychological Review 98(3), 377-389

Huttenlocher, J., P. Smiley and R. Charney

1983 Emergence of action categories in the child: Evidence from verb meanings. Psychological Review 90, 72-93

IMAI, M. AND D. GentNeR

1997 A cross-linguistic study of early word meaning: Universal ontology and linguistic influence. Cognition 62(2), 169-200.

IMAI, M. AND R. MAZUKA

1997 April A crosslinguistic study on the construal of individuation in linguistic and non-linguistic contexts. Paper presented at the Biennial Meeting of the Soceity for Research in Child Development, Washington, DC.

JAKENDOFF, R.

1990 Semantic Structures. Cambridge, MA: MIT Press. 
JONES, N.K.

1991 Development of morphophonemic segments in children's mental representations of words. Applied Psychologinguistics 12, 217-39.

Li, P.W. AND L.R. Gleitman

2002 Turning the tables: language and spatial reasoning. Cognition 83(3), 265-294.

LI, G. AND S. THOMPSON

1981 A Reference Grammar of Mandarin Chinese. California: University of Berkeley Press.

LUCY, J.A.

1992 Language Diversity and Thought: A Reformulation of the Linguistic Relativity Hypothesis. Cambridge, England: Cambridge University Press.

MAGNAMARA, J

1972 Cognitive basis of language learning. Psychological Review 79, 1-13.

Morrow, D.G., G.H. Bower and S.L. GreENSPAN

1990 "Situation-based inferences during narrative comprehension." In A.C. Graesser and G.H. Bower (Eds.), The Psychology of Learning and Motivation: Vol. 25. Inferences and text comprehension (pp. 123-135). San Diego: Academic Press.

Munnich, E., B. Landau and B. Dosher

2001 Spatial language and spatial representation: What is universal? Cognition 81, 171 207.

RATCLIFF, R. AND G. MCKOON

1981 Does activation really spread? Psychological Review 88, 454-462.

RENGANATHAN, V.

1996 Web Assisted Learning and Teaching of Tamil (WALTT). Retrieved May 17, 2002 from http://ccat.sas.upenn.edu/plc/tamilweb

Rosch Heider, E.

1972 Universals in color naming and memory. Fournal of Experimental Psychology 93, $1-20$.

Sera, M.D., J. Gathje and J. Gastillo

1999 Language and ontological knowledge: The contrast between objects and events made by English and Spanish speakers. Fournal of Memory and Language 41, 303326.

SLOBIN, D.I.

1987 Thinking for Speaking. In the Proceedings of the Berkeley Linguistics Society (pp. 435445).

1994 "Passives and alternatives in children's narratives in English, Spanish, German, and Turkish." In B. Fox and P. Hopper (Eds.), Voice: Form and Function (pp. 341-364). Amsterdam/Philadelphia: John Benjamins.

STEEVER, S.B.

1987 "Tamil and the Dravidian Languages." In B. Comrie (Ed.), The World's Major Languages (pp. 725-746). New York: Oxford University Press.

1992 “Tamil.” In W. Bright (Ed.), International Encyclopedia of Linguistics 4 (pp. 131-136). Oxford, England: Oxford University Press. 
TAI, J.H.Y.

1984 "Verbs And Times in Chinese: Vendler's Four Categories." In D. Testen, V. Mishra and J. Drogo (Eds.), Papers from the Parasession on Lexical Semantics (pp. 289-296). Chicago Linguistic Society.

WHORF, B.L.

1956 Language, Thought and Reality: Selected Writings of Benjamin Lee Whorf (Ed. J.B. Carroll). Cambridge, MA: MIT Press.

WAGGOT, V

2000 Chinese characters - a direct link to thought? Chinese language - a direct link to logic? Paper presented at the Linguistic Circle, Department of Theoretical WINER, B.J and Applied Linguistics, University of Edinburgh.

1971 Statistical Principles in Experimental Design. New York: McGraw-Hill, 1971.

YEH, M.

1992 The Stative Situation and the imperfective zhe in Chinese. Texas Linguistic Forum 32: Discourse 231-270.

Author Note

The work reported here was conducted while the first author was in receipt of an Overseas Research Student Award from the committee of Vice-Chancellors and Principals of the Universities of the United Kingdom and a Postgraduate Research Studentship from the Faculty Group of Law and Social Sciences, University of Edinburgh, and forms part of her doctoral thesis at the University of Edinburgh. The authors would like to thank Michael Ramscar for helpful discussions; Ross Alloway, Sarah Haywood, Falk Huettig, Stuart Wilson, David Wilkinson and Roy Welensky for their help with the stimuli used in the experiments; and Karmen Seneviratne and David Packiam for their assistance in data collection. 